\title{
ON SYMMETRIC WORDS IN THE SYMMETRIC GROUP OF DEGREE THREE
}

\author{
ERNEST PŁONKA
}

\begin{abstract}
A word $w\left(x_{1}, x_{2}, \ldots, x_{n}\right)$ from absolutely free group $F_{n}$ is called symmetric $n$-word in a group $G$, if the equality $w\left(g_{1}, g_{2}, \ldots, g_{n}\right)=w\left(g_{\sigma 1}, g_{\sigma 2}, \ldots, g_{\sigma n}\right)$ holds for all $g_{1}, g_{2}, \ldots, g_{n} \in G$ and all permutations $\sigma \in S_{n}$. The set $\mathbf{S}^{(n)}(G)$ of all symmetric $n$-words is a subgroup of $F_{n}$. In this paper the groups of all symmetric 2-words and 3-words for the symmetric group of degree 3 are determined.
\end{abstract}

\section{Introduction}

Let $\mathscr{A}=(A, \mathbf{F})$ be an algebra with a family $\mathbf{F}$ of fundamental finitary operations on $A$ and let $\mathbf{A}^{(n)}(\mathscr{A})$ denote the set of all n-ary polynamials of the algebra $\mathscr{A}$. An element $f \in \mathbf{A}^{(n)}(\mathscr{A})$ is called symmetric if the equality $f\left(a_{\sigma 1}, a_{\sigma 2}, \ldots, a_{\sigma n}\right)=f\left(a_{1}, a_{2}, \ldots, a_{n}\right)$ holds for all $a_{1}, a_{2}, \ldots, a_{n} \in A$ and all permutations $\sigma \in S_{n}$. The set of all symmetric polynomials of $\mathscr{A}$ is denoted by $\mathbf{S}^{(n)}(\mathscr{A})$. In the case of a group $G$ the set $\mathbf{A}^{(n)}(G)$ consists of all functions $G^{n} \ni\left(g_{1}, g_{2}, \ldots, g_{n}\right) \longrightarrow w\left(g_{1}, g_{2}, \ldots, g_{n}\right)$, where $w\left(x_{1}, x_{2}, \ldots, x_{n}\right)$ is a word of $n$ variables, i.e. an element of the free group $\mathscr{F}_{n}$ on free generators $x_{1}, x_{2}, \ldots, x_{n}$. Let $\mathscr{V}_{n}(G)=\mathscr{V}_{n}$ be the subgroup of $\mathscr{F}_{n}$ consisting of all words $w$ such that $w\left(g_{1}, g_{2}, \ldots, g_{n}\right)=1$ for all $g_{1}, g_{2}, \ldots, g_{n} \in G$. For a permutation $\sigma \in S_{n}$ the mapping $x_{i} \longrightarrow x_{\sigma(i)}, 1 \leq i \leq n, \sigma \in S_{n}$, define an automorphism $\varphi_{\sigma}$ of $\mathscr{F}_{n}$. Namely we have $\varphi_{\sigma}(w)\left(x_{1}, x_{2}, \ldots, x_{n}\right)=$ $w\left(x_{\sigma(1)}, x_{\sigma(2)}, \ldots, x_{\sigma(n)}\right)$. Since $\mathscr{V}_{n}$ is complete characteristic subgroup of $\mathscr{F}_{n}$, the automorphism $\varphi_{\sigma}$ induces an automorphism $\bar{\varphi}_{\sigma}$ of the factor group $\mathscr{F}_{n} / \mathscr{\mathscr { V }}_{n}$. Clearly two words $w, v \in \mathscr{F}_{n}$ yield the same element of $\mathbf{A}^{(n)}(G)$ if and only if $w v^{-1} \in \mathscr{V}_{n}$ and therefore the set $\mathbf{S}^{(n)}(G)$ of all $n$-ary symmetric operations in $G$ can be identified with the subgroup of the group $\mathscr{F}_{n} / \mathscr{V}_{n}$ consisting of all elements which are stable under the action of all automorphisms $\bar{\varphi}_{\sigma}, \sigma \in S_{n}$. Thus

$$
\begin{aligned}
\mathbf{S}^{(n)}(G) & =\left\{w \cdot \mathscr{V}_{n} \in \mathscr{F}_{n} / \mathscr{V}_{n}: \bar{\varphi}_{\sigma}\left(w \cdot \mathscr{V}_{n}\right)=w \cdot \mathscr{V}_{n} \text { for all } \sigma \in S_{n}\right\} \\
& =\left\{w \in \mathscr{F}_{n} / \mathscr{V}_{n}: \varphi_{\sigma}(w) \cdot w^{-1} \in \mathscr{V}_{n} \text { for all } \sigma \in S_{n}\right\} .
\end{aligned}
$$

Received November 22, 2004. 
The question of characterization of symmetric words of $n$ variables (shortly $n$-words) in groups was initiated in [11] and [12]. It has been done for arbitrary nilpotent groups of class $\leq 3$ and in the case of symmetric 2-words also for dihedral group of order 2p. In the papers [2] and [9] 2-words are determined for free metabelian groups and soluble groups of derived length 3. A description of the groups $\mathbf{S}^{(2)}(G)$ and $\mathbf{S}^{(3)}(G)$ for free metabelian and free metabelian, nilpotent group $G$ is given in [4]. The same for free nilpotent groups of class 4 and 5 has been done in [5], [6] and [7]. Very recently all symmetric $n$ words for free metabelian groups are characterized in [8]. Some applications of symmetric words one can find in [13]. In this note all symmetric 2-words for the symmetric group $S_{3}$ are listed and using this we determine the group $\mathbf{S}^{(3)}\left(S_{3}\right)$. Unexpectedly enough it turns out that it is isomorphic to the group $\left(Z_{3}\right)^{6}$, whereas the group $\mathbf{S}^{(2)}\left(S_{3}\right)$ is non-Abelian. Moreover we prove that all groups $S^{(n)}\left(S_{3}\right)$ with the exception $n=2$ are commutative.

\section{Preliminaries}

Let us denote $e=(1,2,3), \mathbf{1}=(2,3,1), \mathbf{2}=(3,1,2), \varphi=(2,1,3)$, $\varphi \mathbf{1}=(3,2,1)$ and $\varphi \mathbf{2}=(1,3,2)$. We use standard notation:

$$
x^{-1} y x=y^{x}, \quad x^{-1} y^{-1} x y=[x, y], \quad x^{y+\alpha z+\beta}=x^{y}\left(x^{\alpha}\right)^{z} x^{\beta}, \quad x^{0}=e
$$

for arbitrary group elements $x, y, z$ and all integers $\alpha, \beta$. We often shall make use the following simple

STATEMENTs. (i) The following relations

$$
\begin{aligned}
y x=x y[y, x], \quad[y, x]^{-1} & =[x, y], \quad[x y, z]=[x, z][x, z, y][y, z], \\
{[x, y z] } & =[x, z][x, y][x, y, z]
\end{aligned}
$$

are identities in any group.

(ii) The following equations

$$
\begin{aligned}
x^{6} & =1, \\
{[x, y]^{3} } & =1, \\
{\left[x^{2},[y, z]\right] } & =1, \\
{[[x, y],[z, u]] } & =1
\end{aligned}
$$

are identities in $S_{3}$. The group $S_{3}$ is metabelian (comp. [10]) and therefore the Jacobi identity $J(x, y, z)=1$, i.e. the equality

$$
J(x, y, z)=[y, x]^{1-z}[z, x]^{y-1}[z, y]^{1-x}=1
$$

is an identity in $S_{3}$. 
(iii) For arbitrary group $G$ the relation $s\left(x_{1}, x_{2}, \ldots, x_{n}\right) \in \mathbf{S}^{(n)}(G)$ implies $s\left(x_{1}, x_{2}, \ldots, x_{n-1}, 1\right) \in \mathbf{S}^{(n-1)}(G)$.

(iv) If two words $w, v \in \mathbf{A}^{(2)}\left(S_{3}\right)$ are equal on the pairs $(\mathbf{1}, e),(e, \mathbf{1}),(\mathbf{1}, \varphi)$, $(\varphi, \mathbf{1}),(\varphi, \mathbf{1} \varphi)$ and $(\mathbf{1} \varphi, \varphi)$, then $w(x, y)=v(x, y)$ is an identity in $S_{3}([11$, Lemma]).

\section{Auxiliary results}

We begin with

THEOREM 1. The group $\mathbf{S}^{(2)}\left(S_{3}\right)$ consists of 18 elements

$\begin{array}{lll}1 \cdot 1 \cdot 1=1 & 1 \cdot 1 v=x^{2} y^{2}[y, x]^{x-y} & 1 \cdot 1 v^{2}=x^{4} y^{4}[y, x]^{y-x} \\ s 1 \cdot 1=[y, x]^{x-y} & s 1 v=x^{2} y^{2}[y, x]^{y-x} & s 1 v^{2}=x^{4} y^{4} \\ s^{2} 1 \cdot 1=[y, x]^{y-x} & s^{2} 1 v=x^{2} y^{2} & s^{2} 1 v^{2}=x^{4} y^{4}[y, x]^{x-y} \\ 1 t 1=x^{3} y^{3}[y, x]^{-x-y} & 1 t v=x^{5} y^{5}[y, x]^{1+x+y} & 1 t v^{2}=x y[y, x]^{-1} \\ s t 1=x^{3} y^{3}[y, x]^{x} & s t v=x^{5} y^{5}[y, x]^{1-y} & \text { st } v^{2}=x y[y, x]^{-1-x+y} \\ s^{2} t 1=x^{3} y^{3}[y, x]^{y} & s^{2} t v=x^{5} y^{5}[y, x]^{1-x} & s^{2} t v^{2}=x y[y, x]^{-1+x-y}\end{array}$

where

$$
s=[y, x]^{x-y}, \quad t=x^{3} y^{3}[y, x]^{-x-y} \quad \text { and } \quad v=x^{2} y^{2}[y, x]^{x-y} .
$$

Thus the group $\mathbf{S}^{(2)}\left(S_{3}\right)$ is the direct product of subgroup $\operatorname{gp}\{s, t\} \cong S_{3}$ and cyclic group $\mathrm{gp}\{v\}$ of order 3 .

Proof. It was proved in [11, Theorem 2] that the words $w=x y[y, x]^{-1}$ and $u=x^{2} y^{2}$ are symmetric in the group $S_{3}$ and that the group $\mathbf{S}^{(2)}\left(S_{3}\right)$ is generated by this words. Therefore $s, t$ and $v$ are symmetric words in $S_{3}$. Using the identities from (i) and (ii) one can easily verify the relations $s^{3}=1, t^{2}=1$, $v^{3}=1, s t=t^{2} s, s v=v s$ and $t v=v t$. Hence the group $\operatorname{gp}\{s, t\}$ is isomorphic to $S_{3}$, and thus $\operatorname{gp}\{s, t\} \times\left\{1, v, v^{2}\right\}=\mathbf{S}^{(2)}\left(S_{3}\right)$.

LEMmA 1. If for integers $a, b, c$ and $d$ the equality

$$
[y, x]^{a x+b y+c x y+d}=1
$$

holds for all $x, y \in S_{3}$ then $a \equiv b \equiv c \equiv d(\bmod 3)$.

Proof. We use (iv). Since the equality (5) holds for the pairs $(\mathbf{1}, e),(e, \mathbf{1})$ and all integers $a, b, c$ and $d$, we can restrict ourself to four pairs $(\mathbf{1}, \varphi),(\varphi, \mathbf{1})$, $(b, \mathbf{1} \varphi)$ and $(\mathbf{1} \varphi, \varphi)$. By (iv) the equality (5) is an identity in the group $S_{3}$ if 
and only if the integers $a, b, c$ and $d$ satisfy the following system of equalities

$$
\left\{\begin{array}{l}
\mathbf{2}^{a} \cdot \mathbf{1}^{b} \cdot \mathbf{1}^{c} \cdot \mathbf{2}^{d}=e \\
\mathbf{2}^{a} \cdot \mathbf{1}^{b} \cdot \mathbf{2}^{c} \cdot \mathbf{1}^{d}=e \\
\mathbf{1}^{a} \cdot \mathbf{1}^{b} \cdot \mathbf{2}^{c} \cdot \mathbf{2}^{d}=e \\
\mathbf{2}^{a} \cdot \mathbf{2}^{b} \cdot \mathbf{1}^{c} \cdot \mathbf{1}^{d}=e
\end{array}\right.
$$

Since the mapping $e \longrightarrow 0, \mathbf{1} \longrightarrow 1,2 \longrightarrow 2$ is an isomorphism of the permutation group $\left(\{e, \mathbf{1}, \mathbf{2}\}\right.$; o) onto the cyclic group $Z_{3}$, the last system is equivalent to the homogenous system of equations

$$
\left\{\begin{aligned}
2 a+b+c+2 d & =0 \\
2 a+b+2 c+d & =0 \\
a+b+2 c+2 d & =0 \\
2 a+2 b+c+d & =0
\end{aligned}\right.
$$

where + is taken modulo 3 , of course. Let us observe that vector $(1,1,1,1)$ is a solution of the system. Since the rank of the matrix of the system is 3 , the set $\{(1,1,1,1),(2,2,2,2),(0,0,0,0)\}$ consists of all solutions.

COROLlary 1. The relation

$$
[y, x]^{1+x+y+x y}=1
$$

is an identity in the group $S_{3}$.

COROLlaRY 2. If for some integers $a, b, c$ and $d$ the equality

$$
[y, x]^{a x+b y+c x y+d}=1
$$

holds for all $x, y \in S_{3}$ and at least one from the integers $a, b, c$ or $d$ is 0 $(\bmod 3)$, then all the integers have to be equal $0(\bmod 3)$.

LEMMA 2. The equality

$$
[y, x]^{(1-z)(a+b x+c y+d z)}[z, x]^{f(y-1)(x-y)}=1
$$

holds for all $x, y, z \in S_{3}$ if and only if $c \equiv a-b-d \equiv f-b \equiv 0(\bmod 3)$.

Proof. If we put $z=x$ into (5) then we get

$$
[y, x]^{(a-b-d)+(b+d-a) x+c y-c x y} .
$$


It follows from Lemma 1 that $c \equiv a-b-d \equiv 0(\bmod 3)$. Because of the equality $[y, x]^{(1-z)(1+z)}=1$ we can rewrite $(5)$ as

$$
[y, x]^{b(1-z)(x+1)}[z, x]^{f(y-1)(x-y)}=1 .
$$

By putting $z=y$ and taking into account Lemma 1 we get congruence $f \equiv b$ $(\bmod 3)$.

Conversely, if the congruences hold then the equality (5) is of the form

$$
[y, x]^{b(x+1)(1-z)}[z, x]^{b(x+1)(y-1)}=1,
$$

which is identical with the Jacobi identity $J(x, y, z)^{b(x+1)}=1$.

\section{Symmetric 3-words}

Let $w$ be a word of 3 variables in general form

$$
w=x^{\alpha_{1}} y^{\beta_{1}} z^{\gamma_{1}} x^{\alpha_{2}} y^{\beta_{2}} z^{\gamma_{2}} \cdots x^{\alpha_{n}} y^{\beta_{n}} z^{\gamma_{n}},
$$

where $\alpha_{i}, \beta_{i}, \gamma_{i} \in \mathbf{Z}, i=1,2, \ldots, n$ and $n \in \mathrm{N}$. In view of the identity (1), we may assume that all integers $\alpha_{i}, \beta_{i}$ and $\gamma_{i}$ belong to the set $\{0,1,2,3,4,5\}$. Using the identities from (i) it is possible to remove each $x$ of the word (7) at the first place. One obtains a word of the form $x^{a_{1}} u v \ldots$, where $u, v, \ldots$ are variables $y, z$ or commutators of the form $[y, x]^{x^{i}}$ and $[z, x]^{x^{j}}$ for some $i, j=1,2, \ldots$. Since squares of elements of the group $S_{3}$ commutes with commutators (comp. (3)), one can assume $i$ and $j$ equal 0 or 1 . Now we remove all $y^{\prime} s$ at the second place and apply (ii). We get a word $x^{\alpha} y^{\beta} u^{\prime} v^{\prime} \cdots$, where $u^{\prime}, v^{\prime}, \ldots$ are words of the form $z,[y, x]^{x^{i} y^{j}},[z, x]^{x^{k} y^{l}}$ or $[z, x]^{y^{m}}$ for some $i, j, k, l, m \in\{0,1\}$. Clearly, the same collecting process can be made with the last variable $z$. This together with Corollary 2 gives

Lemma 3. Any word of variables $x, y$ and $z$ in the group $S_{3}$ is equivalent modulo $\mathscr{V}_{3}\left(S_{3}\right)$ to the following word

$$
\begin{aligned}
w(x, y, z)=x^{a} y^{b} z^{c} \cdot[y, x]^{\alpha_{0}+\alpha_{1} x+\alpha_{2} y+\alpha_{3} z+\alpha_{13} x z+\alpha_{23} y z} \\
\cdot[z, x]^{\beta_{0}+\beta_{1} x+\beta_{2} y+\beta_{3} z+\beta_{12} x y+\beta_{23} y z} \\
\cdot[z, y]^{\gamma_{0}+\gamma_{1} x+\gamma_{2} y+\gamma_{3} z+\gamma_{12} x y+\gamma_{13} x z}
\end{aligned}
$$

for some elements $a, b, c \in\{0,1,2,3,4,5\}$ and $\alpha_{0}, \ldots, \gamma_{13} \in\{0,1,2\}$.

From now on we write $w=u$ instead of $w \equiv u\left(\bmod \mathscr{V}_{3}\left(S_{3}\right)\right)$ and we prefer to write -1 than 2 , when 2 is an exponent of a commutator. Now we are able to determine all words of three variables in the group $S_{3}$. First we show 
that the image of each symmetric 3-word in $S_{3}$ treated as a function $S_{3}^{3} \longrightarrow S_{3}$ is contained in the commutator subgroup of $S_{3}$. More precisely we have

THEOREM 2. Let $s(x, y, z)$ be a symmetric 3-word of the form (8) in the group $S_{3}$. Then $a=b=c=2 i$ for $i=0,1$ or 2 .

Proof. Clearly $s(x, 1,1)=s(1, x, 1)=s(x, 1,1)$ and therefore we have $a \equiv b \equiv c(\bmod 6)$.

Let us suppose that $a=1$. We have

$$
\begin{aligned}
& s(x, y, 1)=x y[y, x]^{\left(\alpha_{0}+\alpha_{3}\right)+\left(\alpha_{1}+\alpha_{13}\right) x+\left(\alpha_{2}+\alpha_{23}\right) y}, \\
& s(x, 1, y)=x y[y, x]^{\left(\beta_{0}+\beta_{2}\right)+\left(\beta_{1}+\beta_{12}\right) x+\left(\beta_{3}+\beta_{23}\right) y}, \\
& s(1, x, y)=x y[y, x]^{\left(\gamma_{0}+\gamma_{1}\right)+\left(\gamma_{2}+\gamma_{12}\right) x+\left(\gamma_{3}+\gamma_{13}\right) y} .
\end{aligned}
$$

By (iii) $s(x, y, 1)$ is a symmetric 2-word in $S_{3}$. It follows from Theorem 1 that $s(x, y, 1)$ equals

$$
x y[y, x]^{-1} \quad \text { or } \quad x y[y, x]^{-1-x+y} \quad \text { or } \quad x y[y, x]^{-1+x-y} .
$$

Without loss of generality we can assume that

$$
s(x, y, 1)=x y[y, x]^{-1} .
$$

Indeed, it is easy to verify the equalities $w(x, y, z)=w(y, x, z)=w(x, z, y)$, where

$$
w(x, y, z)=[y, x]^{(y-x) z}[z, x]^{(z-x) y}[z, y]^{(z-y) x},
$$

which means that $w$ is a symmetric 3 -word in the group $S_{3}$ such that $w(x, y, 1)$ $=[y, x]^{y-x}$. Therefore if $s(x, y, 1)$ does not equal $x y[y, x]^{-1}$, then we can consider $s \cdot w$ or $s \cdot w^{2}$ instead of $s$.

By Corollary 2 we get the congruences

$$
\begin{gathered}
\alpha_{0}+\alpha_{3}+1 \equiv \beta_{0}+\beta_{2}+1 \equiv \gamma_{0}+\gamma_{1}+1 \equiv \alpha_{1}+\alpha_{13} \equiv \beta_{1}+\beta_{12} \\
\equiv \gamma_{2}+\gamma_{12} \equiv \alpha_{2}+\alpha_{23} \equiv \beta_{3}+\beta_{23} \equiv \gamma_{3}+\gamma_{13} \equiv 0(\bmod 3)
\end{gathered}
$$

Therefore we can rewrite the word $s$ as

$$
\begin{aligned}
& s(x, y, z)=x y z \cdot[y, x]^{(1-z)\left(\alpha_{0}+\alpha_{1} x+\alpha_{2} y\right)-z} \\
& \cdot[z, x]^{(1-y)\left(\beta_{0}+\beta_{1} x+\beta_{3} z\right)-y} \\
& \cdot[z, y]^{(1-x)\left(\gamma_{0}+\gamma_{2} y+\gamma_{3} z\right)-x} .
\end{aligned}
$$


Now using (i) and (ii) we get

$$
\begin{gathered}
s(y, x, z)=x y z[y, x]^{z} \cdot[y, x]^{(1-z)\left(-\alpha_{0}-\alpha_{2} x-\alpha_{1} y\right)+z} \\
\cdot[z, x]^{(1-y)\left(\gamma_{0}+\gamma_{2} x+\gamma_{3} z\right)-y} \\
\cdot[z, y]^{(1-x)\left(\beta_{0}+\beta_{1} y+\beta_{3} z\right)-x}
\end{gathered}
$$

and similarly

$$
\begin{gathered}
s(x, z, y)=x y z[z, y] \cdot[y, x]^{(1-z)\left(\beta_{0}+\beta_{1} x+\beta_{3} y\right)-z} \\
\cdot[z, x]^{(1-y)\left(\alpha_{0}+\alpha_{1} x+\alpha_{2} z\right)-y} \\
\cdot[z, y]^{(1-x)\left(-\gamma_{0}-\gamma_{3} y-\gamma_{2} z\right)+x} .
\end{gathered}
$$

The condition $s(x, y, z)=s(y, x, z)$ implies

$$
\begin{aligned}
& {[y, x]^{(1-z)\left\{\left(-\alpha_{0}+\left(\alpha_{1}+\alpha_{2}\right) x+\left(\alpha_{1}+\alpha_{2}\right) y\right\}\right.}} \\
& \cdot[z, x]^{(1-y)\left\{\left(\beta_{0}-\gamma_{0}\right)+\left(\beta_{1}-\gamma_{2}\right) x+\left(\beta_{3}-\gamma_{3}\right) z\right\}} \\
& \cdot[z, y]^{(1-x)\left\{\left(\gamma_{0}-\beta_{0}\right)+\left(\gamma_{2}-\beta_{1}\right) y+\left(\gamma_{3}-\beta_{3}\right) z\right\}}=1,
\end{aligned}
$$

which in the case $z=x$ gives

$$
[y, x]^{(1-x)\left\{\left(-\alpha_{0}+\beta_{0}-\gamma_{0}\right)+\left(\alpha_{1}+\alpha_{2}+\beta_{3}-\gamma_{3}\right) x+\left(\alpha_{1}+\alpha_{2}+\beta_{1}-\gamma_{2}\right) y\right\}}=1,
$$

This, by Lemma 2, implies the congruences

$$
\begin{gathered}
\beta_{1} \equiv \gamma_{2}-\alpha_{1}-\alpha_{2}(\bmod 3) \\
-\alpha_{0}+\beta_{0}-\gamma_{0} \equiv \alpha_{1}+\alpha_{2}+\beta_{3}-\gamma_{3}(\bmod 3)
\end{gathered}
$$

Similarly the equality $s(x, y, z)=s(x, z, y)$ gives

$$
\begin{aligned}
& {[y, x]^{(1-z)\left\{\left(\alpha_{0}-\beta_{0}\right)+\left(\alpha_{1}-\beta_{1}\right) x+\left(\alpha_{2}-\beta_{3}\right) y\right\}}} \\
& \cdot[z, x]^{(1-y)\left\{\left(\beta_{0}-\alpha_{0}\right)+\left(\beta_{1}-\alpha_{1}\right) x+\left(\beta_{3}-\alpha_{2}\right) z\right\}} \\
& \cdot[z, y]^{(1-x)\left\{\left(-\gamma_{0}-1+\left(\gamma_{2}+\gamma_{3}\right) y+\left(\gamma_{2}+\gamma_{3}\right) z\right\}\right.}=1,
\end{aligned}
$$

which in the case $y=x$ together with Lemma 2 yields the congruences

$$
\begin{aligned}
\beta_{3} & \equiv \alpha_{2}-\gamma_{2}-\gamma_{3}(\bmod 3) \\
-\alpha_{0}+\beta_{0}-\gamma_{0}-1 & \equiv \beta_{1}-\alpha_{1}+\gamma_{2}+\gamma_{3}(\bmod 3) .
\end{aligned}
$$

After eliminating $\beta_{1}$ and $\beta_{3}$ from above system of four congruences we see that it has no solution.

TheOREM 3. Let $s(x, y, z)$ be a symmetric 3-word in the group $S_{3}$, then (12) $s=u^{i} \cdot s_{0}^{j} \cdot s_{1}^{k} \cdot s_{2}^{l} \cdot s_{3}^{m} \cdot w^{n} \quad$ for some $i, j, k, l, m, n \in\{0,1,-1\}$, 
where

$$
\begin{aligned}
u(x, y, z) & =x^{2} y^{2} z^{2}, \\
s_{0}(x, y, z) & =[y, x]^{z-1}[z, x]^{y-1}, \\
s_{1}(x, y, z) & =[y, x]^{(1-z) x}[z, x]^{1-y}[z, y]^{(1-x)(y-z)}, \\
s_{2}(x, y, z) & =[y, x]^{(1-z) y}[z, x]^{1-y}[z, y]^{(1-x)(y+z)} \\
s_{3}(x, y, z) & =[z, x]^{(1-y)(x+z)}[z, y]^{(1-y)(y+z)}, \\
w(x, y, z) & =[y, x]^{(y-x) z}[z, x]^{(z-x) y}[z, y]^{(z-y) x} .
\end{aligned}
$$

Presentation (12) is unique and therefore $\mathbf{S}^{(3)}\left(S_{3}\right)$ is Abelian group isomorphic to $\left(\mathrm{Z}_{3}\right)^{6}$.

Proof. Let $s(x, y, z)$ be a symmetric 3-word in $S_{3}$ of the form (8) with $a=b=c=0$. We have

$$
\begin{aligned}
& s(x, y, 1)=[y, x]^{\left(\alpha_{0}+\alpha_{3}\right)+\left(\alpha_{1}+\alpha_{13}\right) x+\left(\alpha_{2}+\alpha_{23}\right) y}, \\
& s(x, 1, y)=[y, x]^{\left(\beta_{0}+\beta_{2}\right)+\left(\beta_{1}+\beta_{12}\right) x+\left(\beta_{3}+\beta_{23}\right) y}, \\
& s(1, x, y)=[y, x]^{\left(\gamma_{0}+\gamma_{1}\right)+\left(\gamma_{2}+\gamma_{12}\right) x+\left(\gamma_{3}+\gamma_{13}\right) y} .
\end{aligned}
$$

By Theorem 1 every symmetric word $s(x, y, 1)$ of two variables equals

$$
\text { either } 1 \text { or }[y, x]^{y-x} \text { or else }[y, x]^{x-y} \text {. }
$$

Let us consider first case $s(x, y, 1)=1$.

By Corollary 2 we have the following congruences

$$
\begin{aligned}
\alpha_{0}+\alpha_{3} & \equiv \beta_{0}+\beta_{2} \equiv \gamma_{0}+\gamma_{1} \equiv \alpha_{1}+\alpha_{13} \equiv \beta_{1}+\beta_{12} \\
& \equiv \gamma_{2}+\gamma_{12} \equiv \alpha_{2}+\alpha_{23} \equiv \beta_{3}+\beta_{23} \equiv \gamma_{3}+\gamma_{13} \equiv 0(\bmod 3)
\end{aligned}
$$

which enables us to rewrite the word $s$ in the form

$$
\begin{aligned}
s(x, y, z)=[ & y, x]^{(1-z)\left(\alpha_{0}+\alpha_{1} x+\alpha_{2} y\right)} \\
& \cdot[z, x]^{(1-y)\left(\beta_{0}+\beta_{1} x+\beta_{3} z\right)} \\
& \cdot[z, y]^{(1-x)\left(\gamma_{0}+\gamma_{2} y+\gamma_{3} z\right)} .
\end{aligned}
$$

It is well known the transpositions $(1,2)$ and $(2,3)$ generate the symmetric group $S_{3}$ of degree 3 and therefore $s(x, y, z)$ is symmetric if and only if two equalities

$$
s(y, x, z)^{-1} \cdot s(x, y, z)=1, \quad s(x, z, y)^{-1} \cdot s(x, y, z)=1
$$


hold for all elements $x, y, z$ from $S_{3}$. We check

$$
\begin{gathered}
s(y, x, z)=[y, x]^{(1-z)\left(-\alpha_{0}-\alpha_{2} x-\alpha_{1} y\right)} \cdot[z, x]^{(1-y)\left(\gamma_{0}+\gamma_{2} x+\gamma_{3} z\right)} \\
\cdot[z, y]^{(1-x)\left(\beta_{0}+\beta_{1} y+\beta_{3} z\right)}
\end{gathered}
$$

and similarly

$$
\begin{gathered}
s(x, z, y)=[y, x]^{(1-z)\left(\beta_{0}+\beta_{1} x+\beta_{3} y\right)} \cdot[z, x]^{(1-y)\left(\alpha_{0}+\alpha_{1} x+\alpha_{2} z\right)} \\
\cdot[z, y]^{(1-x)\left(-\gamma_{0}-\gamma_{3} y-\gamma_{2} z\right)} .
\end{gathered}
$$

Hence we get

$$
\begin{aligned}
& f(x, y, z)=s(y, x, z)^{-1} \cdot s(x, y, z) \\
&=[y, x]^{(1-z)\left\{-\alpha_{0}+\left(\alpha_{1}+\alpha_{2}\right) x+\left(\alpha_{1}+\alpha_{2}\right) y\right\}} \\
& \cdot[z, x]^{(y-1)\left\{\left(\gamma_{0}-\beta_{0}\right)+\left(\gamma_{2}-\beta_{1}\right) x+\left(\gamma_{3}-\beta_{3}\right) z\right\}} \\
& \cdot[z, y]^{(1-x)\left\{\left(\gamma_{0}-\beta_{0}\right)+\left(\gamma_{2}-\beta_{1}\right) y+\left(\gamma_{3}-\beta_{3}\right) z\right\}}
\end{aligned}
$$

and also

$$
\begin{aligned}
& g(x, y, z)=s(x, z, y)^{-1}(s(x, y, z) \\
&=[y, x]^{(1-z)\left\{\left(\alpha_{0}-\beta_{0}\right)+\left(\alpha_{1}-\beta_{1}\right) x+\left(\alpha_{2}-\beta_{3}\right) y\right\}} \\
& \cdot[z, x]^{(y-1)\left\{\left(\alpha_{0}-\beta_{0}\right)+\left(\alpha_{1}-\beta_{1}\right) x+\left(\alpha_{2}-\beta_{3}\right) z\right\}} \\
& \cdot[z, y]^{(1-x)\left\{-\gamma_{0}+\left(\gamma_{2}+\gamma_{3}\right) y+\left(\gamma_{2}+\gamma_{3}\right) z\right\}}
\end{aligned}
$$

Thus $s$ is symmetric if and only if the equalities $f(x, y, z)=g(x, y, z)=1$ hold for all $x, y, z \in S_{3}$. Applying Jacobi identity

$$
\left([y, x]^{1-z}[z, x]^{y-1}[z, y]^{1-x}\right)^{\left\{\left(\gamma_{0}-\beta_{0}\right)+\left(\gamma_{2}-\beta_{1}\right) y+\left(\gamma_{3}-\beta_{3}\right) z\right\}}=1
$$

to the equality (14) and

$$
\left([y, x]^{1-z}[z, x]^{y-1}[z, y]^{1-x}\right)^{\left\{\left(\alpha_{0}-\beta_{0}\right)+\left(\alpha_{1}-\beta_{1}\right) x+\left(\alpha_{2}-\beta_{3}\right) y\right\}}=1
$$

to the equality (15) we see that $s(x, y, z)$ is symmetric 3-word if and only if the following two equalities

$$
\begin{gathered}
1=f(x, y, z)=[y, x]^{(1-z)\left\{\left(\beta_{0}-\alpha_{0}-\gamma_{0}\right)+\left(\alpha_{1}+\alpha_{2}\right) x+\left(\alpha_{1}+\alpha_{2}+\beta_{1}-\gamma_{2}\right) y+\left(\beta_{3}-\gamma_{3}\right) z\right\}} \\
\cdot[z, x]^{\left(\gamma_{2}-\beta_{1}\right)(y-1)(x-y)}, \\
1=g(x, y, z)=[z, y]^{(1-x)\left\{\left(\beta_{0}-\alpha_{0}-\gamma_{0}\right)+\left(\beta_{1}-\alpha_{1}\right) x+\left(\gamma_{2}+\gamma_{3}+\beta_{3}-\alpha_{2}\right) y+\left(\gamma_{2}+\gamma_{3}\right) z\right\}} \\
\cdot[z, x]^{\left(\alpha_{2}-\beta_{3}\right)(y-1)(z-y)}
\end{gathered}
$$


holds for all $x, y, z \in S_{3}$. By Lemma 2 this is equivalent to the following system of congruences

$$
\begin{gathered}
\alpha_{1}+\alpha_{2}+\beta_{1}-\gamma_{2} \equiv 0(\bmod 3), \\
-\alpha_{0}+\beta_{0}-\gamma_{0} \equiv \alpha_{1}+\alpha_{2}+\beta_{3}-\gamma_{3}(\bmod 3), \\
\beta_{3}-\gamma_{3} \equiv \gamma_{2}-\beta_{1}(\bmod 3), \\
-\alpha_{2}+\beta_{3}+\gamma_{2}+\gamma_{3} \equiv 0(\bmod 3), \\
-\alpha_{0}+\beta_{0}-\gamma_{0} \equiv \beta_{1}-\alpha_{1}+\gamma_{2}+\gamma_{3}(\bmod 3) .
\end{gathered}
$$

Choosing $\alpha_{0}, \gamma_{0}, \alpha_{1}, \alpha_{2}$ and $\beta_{1}$ as parameters we obtain the following solution of the system

\begin{tabular}{|c|c|c|c|c|}
\hline & 0 & 1 & 2 & 3 \\
\hline$\alpha$ & $\alpha_{0}$ & $\alpha_{1}$ & $\alpha_{2}$ & $-\alpha_{0}$ \\
\hline$\beta$ & $\alpha_{0}+\gamma_{0}-\alpha_{1}-\alpha_{2}$ & $\beta_{1}$ & $\alpha_{0}-\gamma_{0}+\alpha_{1}+\alpha_{2}$ & $\beta_{1}-\alpha_{2}$ \\
\hline$\gamma$ & $\gamma_{0}$ & $-\gamma_{0}$ & $\alpha_{1}+\alpha_{2}+\beta_{1}$ & $-\alpha_{1}+\alpha_{2}+\beta_{1}$ \\
\hline
\end{tabular}

Thus the word $s$ can be written as

$$
\begin{gathered}
s(x, y, z)=[y, x]^{(1-z)\left(\alpha_{0}+\alpha_{1} x+\alpha_{2} y\right)} \cdot[z, x]^{(1-y)\left(\left(\alpha_{0}+\gamma_{0}-\alpha_{1}-\alpha_{2}\right)+\beta_{1} x+\left(\beta_{1}-\alpha_{2}\right) z\right.} \\
\cdot[z, y]^{(1-x)\left(\gamma_{0}+\left(\alpha_{1}+\alpha_{2}+\beta_{1}\right)(y-z)\right)},
\end{gathered}
$$

or

$$
s=s_{0}^{\alpha_{0}} \cdot s_{4}^{\gamma_{0}} \cdot s_{1}^{\alpha_{1}} s_{2}^{\alpha_{2}} \cdot s_{3}^{\beta_{1}},
$$

where

$$
\begin{aligned}
& s_{0}(x, y, z)=[y, x]^{z-1}[z, x]^{y-1}, \\
& s_{4}(x, y, z)=[z, x]^{y-1}[z, y]^{x-1}, \\
& s_{1}(x, y, z)=[y, x]^{(1-z) x}[z, x]^{(1-y)}[z, y]^{(1-x)(y-z)}, \\
& s_{2}(x, y, z)=[y, x]^{(1-z) y}[z, x]^{1-y}[z, y]^{(1-x)(y+z)}, \\
& s_{3}(x, y, z)=[z, x]^{(1-y)(x+z)}[z, y]^{(1-x)(y+z)} .
\end{aligned}
$$

Above we have made use of (i) and (ii). Observe that

$$
s_{0}(x, y, z) \cdot s_{4}(x, y, z)=J(x, y, z)^{-1}=1,
$$

which yields

$$
s=s_{0}^{j} \cdot s_{1}^{k} \cdot s_{2}^{l} \cdot s_{3}^{m}
$$


for some $j, k, l, m \in\{0,1,-1\}$. We claim that the presentation (16) of the word $s$ is unique. Indeed, if for some $j, k, l, m \in\{0,1,-1\}$ the equality

$$
s_{0}(x, y, z)^{j} s_{1}(x, y, z)^{k} s_{2}(x, y, z)^{l} s_{3}(x, y, z)^{m}=1
$$

hold for all $x, y, z \in S_{3}$, then, in the case $z=x$, we get

$$
[y, x]^{\{(-j+k+l+m)+(j-k-l-m) x-(k+m) y+(k+m) x y)\}}=1,
$$

which by Lemma 1 implies $l-j \equiv k+m \equiv 0(\bmod 3)$. If we put $z=y$ into (16), we get

$$
[y, x]^{(j+k-m)+(j+k+m) x+(j-k+m) y-(k+m) x y}=1 .
$$

By Corollary 2 we have $j=k=l=m=0$, as required.

As we have mentioned earlier $u=x^{2} y^{2} z^{2}$ is symmetric 3-word in $S_{3}$ and

$$
w(x, y, z)=[y, x]^{(y-x) z}[z, x]^{(z-x) y}[z, y]^{(z-y) x}
$$

is a symmetric 3-word with $w(x, y, 1)=[y, x]^{y-x}$. Let $s(x, y, z)$ be arbitrary symmetric 3-word in $S_{3}$ such that $s(x, y, 1)=\left(x^{2} y^{2}\right)^{i}[y, x]^{n(y-x)}$ for some $i, n=0,1,-1$. Then the following product $\left(u^{-i} s w^{-n}\right)$ is a symmetric 3-word with $\left(u^{-i} s w^{-n}\right)(x, y, 1)=1$ and therefore, in view of what we have just established, $u^{-i} s w^{-n}=s_{0}^{j} s_{1}^{k} s_{2}^{l} s_{3}^{m}$ for some $j, k, l, m, n$, which completes the proof.

THEOREM 4. For all $n \neq 2$ the groups $\mathbf{S}^{(n)}\left(S_{3}\right)$ of $n$-symmetric words of the group $S_{3}$ are commutative.

Proof. Let $s\left(x_{1}, x_{2}, \ldots, x_{n}\right)$ be symmetric n-word, $n \geq 3$, in $S_{3}$. Using the same arguments as in the proof of Theorem 1 it is possible to present the word $s$ as $x_{1}^{a_{1}} x_{2}^{a_{2}}, \ldots, x_{n}^{a_{n}} c$, where $c$ is a product of commutators of the form

$$
\left[x_{i_{1}}, x_{i_{2}}\right]^{P}
$$

for $P$ being a polynomial in variables $x_{1}, x_{2}, \ldots, x_{n}$. Since $s(x, 1,1, \ldots, 1)=$ $s(1, x, 1, \ldots, 1)=\cdots=s(1,1, \ldots, 1, x)$, we have the equality $a_{1} \equiv a_{2} \equiv$ $\ldots \equiv a_{n} \equiv a(\bmod 6)$. In view of statement (iii) $s(x, y, z, 1, \ldots, 1)=$ $x^{a} y^{a} z^{a} c^{\prime}$ is a symmetric 3-word in $S_{3}$. By Theorem 2, the number $a$ has to be even, which together with (2) of (ii) finishes the proof.

REMARK. Every symmetric $n$-word in a group $G$ is symmetric in any group from the variety $\operatorname{var}(G)$ of groups generated by $G$ and therefore the results of the paper are valid not only for the group $S_{3}$ but also for all groups from $\operatorname{var}\left(S_{3}\right)=H S P\left(S_{3}\right)$. 


\section{REFERENCES}

1. Gupta, C. K., Hołubowski, W., On the linearity of free nilpotent-by-abelian groups, J. Algebra 24 (1993), 293-302.

2. Gupta, C. K., Hołubowski, W., On 2-symmetric words for groups, Arch. Math. 73 (1999), 327-331.

3. Gupta, C. K., Hołubowski, W., On 2-symmetric words in nilpotent groups, Ukrainian Math. J. 54 (2002), 1020-1024.

4. Hołubowski, W., Symmetric words in metabelian groups, Comm. Algebra 23 (1995), 51615167.

5. Hołubowski, W., Symmetric words in free nilpotent groups of class 4, Publ. Math. Debrecen 57 (2000), 411-419.

6. Hołubowski, W., Symmetric words in nilpotent groups of class 5, London Math. Soc. Lecture Note Ser. 260 (1999), 363-367.

7. Hołubowski, W., Symmetric words in free nilpotent groups of class 4, Publ. Math. Debrecen 57 (2000), 411-419.

8. Hołubowski, W., Note on symmetric words in metabelian groups, Ukrainian Math. J. 54 (2002), 1013-1015.

9. Macedonska, O., Solitar, D., On binary $\sigma$-invariant words in a group, Contemp. Math. 169 (1994), 431-449.

10. Magnus, W., Karrass, A., Solitar, D., Combinatorial group theory: Presentations of Groups in Terms of Generators and Relations, Intersciences Publishers, 1966.

11. Płonka, E., Symmetric operations in groups, Colloq. Math. 21 (1970), 179-186.

12. Płonka, E., Symmetric operations in nilpotent groups of class $\leq$ 3, Fund. Math. 97 (1977), 95-103.

13. Płonka E., On a non-Abelian variety of groups which are symmetric algebras, Math. Scand. 74 (1994), 184-190.

INSTITUTE OF MATHEMATICS

SILESIAN UNIVERSITY OF TECHNOLOGY

UL. KASZUBSKA 23

44-100 GLIWICE

POLAND

E-mail: eplonka@polsl.pl 